\title{
A fully coupled constitutive model for thermo-hydro-mechanical analysis in elastic media with double porosity
}

\author{
N. Khalili ${ }^{1}$ and A. P. S. Selvadurai ${ }^{2}$ \\ Received 15 October 2003; revised 11 November 2003; accepted 13 November 2003; published 23 December 2003.
}

[1] The partial differential equations governing nonisothermal flow and deformation in an elastic medium with double porosity are presented. The governing equations satisfy an effective stress concept, Darcy's law, Fourier' law and the equations of Hookean thermoelasticity. The equations are derived using a systematic macroscopic approach that satisfies conservation laws applicable to balance of linear momentum, mass and energy. The Thermo-Hydro-Mechanical coupling take into account processes associated with: thermal expansion, thermal convection by moving fluid, fluid flux due to temperature gradients, heat flux due to pressure gradients, fluid and heat exchange between the two pore system, and the heat of phase compression. INDEX TERMS: 5104 Physical Properties of Rocks: Fracture and flow; 5114 Physical Properties of Rocks: Permeability and porosity; 5134 Physical Properties of Rocks: Thermal properties; 5199 Physical Properties of Rocks: General or miscellaneous; 9810 General or Miscellaneous: New fields (not classifiable under other headings). Citation: Khalili N., and A. P. S. Selvadurai, A fully coupled constitutive model for thermo-hydro-mechanical analysis in elastic media with double porosity, Geophys. Res. Lett., 30(24), 2268, doi:10.1029/ 2003GL018838, 2003.

\section{Introduction}

[2] The thermo-hydro-mechanical (THM) coupling, influencing heat and mass transport in deformable porous media has received increasing attention in various branches of science and engineering, primarily due to its importance to processes such as secondary enhancement of oil production, storage of thermal energy in the form of sensible and latent heat, remediation of contaminated sites, isolation of hazardous waste, etc. THM processes are also of interest to applications related to insulation materials, heat emitting buried cable systems, tumour treatment, and in the cooling of microelectronic components. Constitutive models have been developed over the past two decades in relation to topics such as coupled heat and moisture transfer in agricultural engineering and in the study of THM processes in natural and engineered barriers for the storage of heat emitting nuclear fuel waste. In spite of their importance in the fields of petroleum engineering, reservoir engineering, and

\footnotetext{
${ }^{1}$ School of Civil and Environmental Engineering, The University of New South Wales, Sydney, Australia.

${ }^{2}$ Department of Civil Engineering and Applied Mechanics, McGill University, Montreal, QC, Canada.
}

Copyright 2003 by the American Geophysical Union. 0094-8276/03/2003GL018838\$05.00 nuclear waste management, THM coupling effects in media with double porosity have rarely been investigated [Bai and Roegiers, 1994; Masters et al., 2000].

[3] In this paper, the governing equations for THM processes in an elastic medium with double porosity are derived using a systematic macroscopic approach, that satisfies appropriate constitutive and conservation laws. The comprehensive array of THM processes taken into account, include: thermo-mechanical deformations, fluid transport, heat transfer, fluid flux due to temperature gradients, heat flux due to pressure gradients, fluid and heat exchange between the double pore system, heat transfer due to fluid movement, and the presence of heat of phase compression. Three phases (solid, pore fluid and fissure fluid) and two constituents (solid and liquid) are identified. Each phase is viewed as an independent continuum, endowed with its own kinematics, mass, momentum and energy. Both solid and fluid constituents are assumed slightly compressible, but if required, either can be made incompressible. Matrix displacement vector, pore fluid pressure, fissure fluid pressure, solid temperature, pore fluid temperature and fissure fluid temperature are introduced as the primary variables.

\section{The Conceptual Model}

[4] The formulation presented consists of three separate, yet inter-connected, models involving (1) deformation, (2) flow model, and (3) heat transfer. The deformation model is based on the classical theory of thermoelasticity, the effective stress concept and the equations of equilibrium. The fluid flow through the medium is modelled using the double porosity concept. Two interacting continua are identified: one representing the porous blocks and the other the fissure network. The two flow regions are connected through a leakage term, describing transfer of fluid from the porous blocks into the fissure network, and vice versa. The rate of fluid transfer at any point depends upon the fluid pressure difference between the porous blocks and the fissure network, as well as the permeability of the porous blocks and the fissure spacing. The coupling between the flow and the deformation fields is established through the effective stress parameters, $\zeta_{1}$ and $\zeta_{2}$, which relate pore and fissure fluid pressures to matrix deformation vector. The heat transfer model is based on the assumption of thermal non-equilibrium between the phases. At any material point, three temperatures are introduced; one for each of the solid, the pore fluid and the fissure fluid phases. The modes of heat transfer are assumed to be: conduction, advection, and heat exchange. The thermal interaction between the phases is established through heat exchange terms. The coupling between the heat transfer model and the flow and the 
deformation models is established through thermal expansion coefficients and the heat of phase compression. In the case of the flow model, an additional coupling is also established through the thermal energy transfer due to the leakage term.

\section{The Deformation Model}

[5] To formulate the deformation model, we define the effective stress in the solid skeleton of a doubly porous medium, $\sigma_{i j}^{\prime}$, as [Khalili and Valliappan, 1996],

$$
\sigma_{i j}=\sigma_{i j}^{\prime}-\zeta_{1} p_{1} \delta_{i j}-\zeta_{2} p_{2} \delta_{i j} ; \quad i, j=1,2,3
$$

where $\sigma_{i j}$ is the total stress and $p_{\alpha}$ is the fluid pressure (positive for compressive pressure and negative for tensile or suction), with subscripts $\alpha=1$ and $\alpha=2$ referring to the porous blocks and fissure network, respectively, and, $\delta_{i j}$ is the Kronecker's delta function. The effective stress parameters, $\zeta_{1}$ and $\zeta_{2}$, are defined as [Khalili and Valliappan, 1996; Loret and Rizzi, 1999; Callari and Federico, 2000; Pao and Lewis, 2002],

$$
\zeta_{1}=\left(c_{p}-c_{s}\right) / c \text { and } \zeta_{2}=\left(c-c_{p}\right) / c
$$

where $c_{S}$ is the elastic compressibility of solid constituent, $c$ is the underlying elastic drained compressibility of the fissured porous media, and $c_{p}$ is the elastic drained compressibility of the porous blocks. Assuming, the compressibility of solid grains to be negligible, Equation 2 yields $\zeta_{1}+\zeta_{2}=1$. Notice that in the limiting case of zero fissure volume we have $c_{p}=c$ thus, $\zeta_{1}=1$ and $\zeta_{2}=0$, and Equation 2 reduces to that proposed by Terzaghi for saturated elastic medium with a single porosity. The Duhamel-Neumann extension to Hooke's law gives the thermoelastic constitutive relationship as,

$$
\sigma_{i j}^{\prime}=2 G \varepsilon_{i j}+\left(\lambda \varepsilon_{k k}-\gamma T_{s}\right) \delta_{i j}
$$

where $G$ and $\lambda$ are the Lamé constants, $T_{s}$ is the temperature of the solid constituent, and $\gamma \delta_{i j}=-\left.\left(\partial \sigma_{i j}^{\prime} / \partial T_{s}\right)\right|_{\varepsilon_{k \cdot}}$ and $\gamma$ is related to the volumetric thermal expansion of the fissured porous medium, $c_{T}$, through $\gamma=\left(c_{T} / c\right)$, with $c=(2 G / 3+$ $\lambda)^{-1}$. Notice that in Equation (3) the macroscopic thermal expansion of the fractured porous medium is defined in terms of the temperature of the solid constituent only rather than an average of the solid and the pore fluid temperatures. This is an important aspect, which is often ill-posed in the literature.

[6] The strain $\varepsilon_{i j}$ is the related to the displacement components, $u_{i}$, through

$$
\varepsilon_{i j}=\frac{1}{2}\left(\frac{\partial u_{i}}{\partial x_{j}}+\frac{\partial u_{j}}{\partial x_{i}}\right)
$$

Considering the equations of equilibrium,

$$
\frac{\partial \sigma_{i j}}{\partial x_{j}}+F_{i}=0
$$

and combining it with Equations 1 to 4 yields,

$$
\frac{\partial}{\partial x_{j}}\left[G \frac{\partial u_{i}}{\partial x_{j}}+(\lambda+G) \frac{\partial u_{j}}{\partial x_{i}}-\left(\zeta_{1} p_{1}+\zeta_{2} p_{2}+\gamma T_{s}\right) \delta_{i j}\right]+F_{i}=0
$$

in which $F_{i}$ is ith component of the body force per unit volume. The set of partial differential Equations 6, for govern the deformation phenomenon in a thermoelastic double porous medium.

\section{The Flow Model}

[7] The flow model is obtained by combining the equations of linear momentum and mass balance for the liquid phase. Neglecting inertial effects, the equation of linear momentum balance can be written as,

$$
v_{\alpha i}^{r}=-\frac{k_{\alpha i j}}{\mu_{\alpha}}\left(\frac{\partial p_{\alpha}}{\partial x_{j}}+\rho_{\alpha} g_{j}\right)-k_{\alpha T i j} \frac{\partial T_{\alpha}}{\partial x_{j}}
$$

where $k_{\mathrm{\alpha} i j}$ is the intrinsic permeability and $k_{\alpha T i j}$ is the coefficient of thermal coupling for fluid flux, analogous to the Soret effect in molecular diffusion, in which mass flux is caused due to both a concentration gradient and a temperature gradient. Also, $\mu_{\alpha}$ and $\rho_{\alpha}$ are the dynamic viscosity and the density of fluid respectively, $g_{i}$ is the $i t h$ component of gravitational acceleration, and $v_{\mathrm{\alpha} i}^{r}$ is the relative velocity of the fluid defined as,

$$
v_{\alpha i}^{r}=n_{\alpha}\left(v_{\alpha i}-v_{s i}\right)
$$

where $n_{\alpha}$ is the porosity, $v_{\alpha i}$ is the absolute fluid velocity and $v_{s i}$ is the solid skeleton velocity,

$$
v_{\alpha i}=\frac{\partial u_{\alpha i}}{\partial t} \text { and } v_{s i}=\frac{\partial u_{i}}{\partial t}
$$

where $u_{\mathrm{\alpha} i}$ and $u_{i}$ are the components of displacements of fluid and solid, respectively.

[8] For conservation of fluid mass, we require

$$
-\frac{\partial}{\partial x_{i}}\left(\rho_{\alpha} n_{\alpha} v_{\alpha i}\right)=\frac{\partial}{\partial t}\left(n_{\alpha} \rho_{\alpha}\right)+(-1)^{\alpha+1} \rho_{\alpha} \Gamma
$$

where $\Gamma$ is the leakage term controlling the rate of fluid transfer between the fissure network and the porous blocks, defined as,

$$
\Gamma=\eta\left(p_{1}-p_{2}\right)
$$

in which $\eta$ is the leakage parameter. Substituting Equation 8 into 10 , we obtain

$$
-\frac{\partial}{\partial x_{i}}\left(\rho_{\alpha} v_{\alpha i}^{r}\right)-\frac{\partial}{\partial x_{i}}\left(\rho_{\alpha} n_{\alpha} v_{s i}\right)=\frac{\partial}{\partial t}\left(\rho_{\alpha} n_{\alpha}\right)+(-1)^{\alpha+1} \rho_{\alpha} \Gamma
$$

and introducing the Lagrangian total derivative concept with respect to the moving solids, $d_{s}(.) / d t=\partial(.) / \partial t+v_{s i} \partial($. 
$\partial x_{i}$, and the moving fluid, $d_{l}(.) / d t=\partial(.) / \partial t+v_{l i} \partial(.) / \partial x_{i}$, Equation 12 can be rearranged to,

$$
-\rho_{\alpha} \frac{\partial}{\partial x_{i}}\left(v_{\alpha i}^{r}\right)=n_{\alpha} \frac{d_{\alpha} \rho_{\alpha}}{d t}+\rho_{\alpha} \frac{d_{s} n_{\alpha}}{d t}+\rho_{\alpha} n_{\alpha} \frac{\partial v_{s i}}{\partial x_{i}}+(-1)^{\alpha+1} \rho_{\alpha} \Gamma
$$

Considering a barotropic fluid $\rho_{\alpha}=\rho_{\alpha}\left(p_{\alpha}, T_{\alpha}\right)$,

$$
\frac{d_{\alpha} \rho_{\alpha}}{d t}=\rho_{\alpha} c_{\alpha} \frac{d_{\alpha} p_{\alpha}}{d t}-\rho_{\alpha} c_{\alpha T} \frac{d_{\alpha} T_{\alpha}}{d t}
$$

in which $c_{\alpha}=\left.\frac{1}{\rho_{\alpha}} \frac{\partial \rho_{\alpha}}{\partial p_{\alpha}}\right|_{T_{\alpha}}$ and $c_{\alpha T}=-\left.\frac{1}{\rho_{\alpha}} \frac{\partial \rho_{\alpha}}{\partial T_{\alpha}}\right|_{p_{\alpha}}$ are the coefficients of compressibility and thermal expansion for fluid, respectively. Noting $n_{\alpha}=\frac{V_{\alpha}}{V}$,

$$
\frac{d_{s} n_{\alpha}}{d t}=\frac{1}{V}\left(\frac{d_{s} V_{\alpha}}{d t}-n_{\alpha} \frac{d_{s} V}{d t}\right)
$$

and assigning $\alpha=1,2$, Equations 7, 13, 14 and 15 can be combined to give

$$
\begin{aligned}
\frac{\partial}{\partial x_{i}}\left[\frac{k_{1 i j}}{\mu_{1}}\left(\frac{\partial p_{1}}{\partial x_{j}}+\rho_{1} g_{j}\right)+k_{1 T i j} \frac{\partial T_{1}}{\partial x_{j}}\right]= & n_{1} c_{1} \frac{d_{1} p_{1}}{d t}+\frac{1}{V} \frac{d_{s} V_{1}}{d t} \\
& -n_{1} c_{1 T} \frac{d_{1} T_{1}}{d t}+\Gamma
\end{aligned}
$$

$$
\begin{aligned}
\frac{\partial}{\partial x_{i}}\left[\frac{k_{2 i j}}{\mu_{2}}\left(\frac{\partial p_{2}}{\partial x_{j}}+\rho_{2} g_{j}\right)+k_{2} T_{i j} \frac{\partial T_{2}}{\partial x_{j}}\right]= & n_{2} c_{2} \frac{d_{2} p_{2}}{d t}+\frac{1}{V} \frac{d_{s} V_{2}}{d t} \\
& -n_{2} c_{2 T} \frac{d_{2} T_{2}}{d t}-\Gamma
\end{aligned}
$$

In Equations 16, $\frac{d_{S} V_{1}}{V}$ and $\frac{d_{s} V_{2}}{V}$ represent the change in the pore and fissure volume over the current volume of the porous medium with respect to the moving solid. For isothermal conditions, $\frac{d_{s} V_{1}}{V}$ and $\frac{d_{s} V_{2}}{V}$ can be calculated from [Khalili and Valliappan, 1996],

$$
\begin{aligned}
\frac{d_{s} V_{1}}{V}= & \left(c_{p}-c_{s}\right) d_{s} \frac{\sigma_{i i}}{3}+\left[\left(1-n_{2}\right) c_{p}-\left(1+n_{1}-n_{2}\right) c_{s}\right] d_{s} p_{1} \\
& +n_{2}\left(c_{p}-c_{s}\right) d_{s} p_{2}
\end{aligned}
$$

$$
\frac{d_{s} V_{2}}{V}=\left(c-c_{p}\right) d_{s} \frac{\sigma_{i i}}{3}+n_{2}\left(c_{p}-c_{s}\right) d_{s} p_{1}+\left[c-\left(1+n_{2}\right) c_{p}\right] d_{s} p_{2}
$$

Extending Equations 17 to include thermal effects, we have,

$$
\begin{aligned}
\frac{d_{s} V_{1}}{V}= & \left(c_{p}-c_{s}\right) d_{s} \frac{\sigma_{i i}}{3}+\left[\left(1-n_{2}\right) c_{p}-\left[\left(1+n_{1}-n_{2}\right) c_{s}\right] d_{s} p_{1}\right. \\
& +n_{2}\left(c_{p}-c_{s}\right) d_{s} p_{2}+n_{1} c_{T} d_{s} T_{s}
\end{aligned}
$$

$$
\begin{aligned}
\frac{d_{s} V_{2}}{V}= & \left(c-c_{p}\right) d_{s} \frac{\sigma_{i i}}{3}+n_{2}\left(c_{p}-c_{s}\right) d_{s} p_{1}+\left[c-\left(1+n_{2}\right) c_{p}\right] d_{s} p_{2} \\
& +n_{2} c_{T} d_{s} T_{s}
\end{aligned}
$$

The last terms on the left hand side of $18 \mathrm{a}$ and $18 \mathrm{~b}$ represent the change in the pore and fissure volume due to a change in the volume of the fissured porous medium with solid temperature, respectively.

[9] Now, using Equations 1 to 3, Equation 18 can be rearranged to,

$$
\begin{aligned}
\frac{d_{s} V_{1}}{V}=\zeta_{1} d_{s} \varepsilon_{i i}+\left[\left(\zeta_{2}-n_{2}\right)\left(c_{p}-c_{s}\right)+\left(\zeta_{1}-n_{1}\right) c_{s}\right] d_{s} p_{1} \\
-\left(\zeta_{2}-n_{2}\right)\left(c_{p}-c_{s}\right) d_{s} p_{2}-\left(\zeta_{1}-n_{1}\right) c_{T} d_{s} T_{s} \\
\frac{d_{s} V_{2}}{V}=\zeta_{2} d_{s} \varepsilon_{i i}+\left(\zeta_{2}-n_{2}\right) c_{p} d_{s} p_{2}-\left(\zeta_{2}-n_{2}\right)\left(c_{p}-c_{s}\right) d_{s} p_{1} \\
\quad-\left(\zeta_{2}-n_{2}\right) c_{T} d_{s} T_{s}
\end{aligned}
$$

Substituting (19) into (16), noting that $\frac{1}{V} \frac{d_{s} V}{d t}=\frac{\partial v_{s i}}{\partial x_{i}}$, and introducing the approximations $v_{\alpha} \frac{\partial p_{\alpha}}{\partial x_{i}} \ll \frac{\partial p_{\alpha}}{\partial t}, v_{s i} \frac{\partial(.)}{\partial x_{i}} \ll \frac{\partial(.)}{\partial t}$ or $\frac{d_{\alpha} p_{\alpha}}{d t} \approx \frac{\partial p_{\alpha}}{\partial t}, \frac{d_{s}(.)}{d t} \approx \frac{\partial(.)}{\partial t}$, yield the differential equations governing non-isothermal fluid flow through fissured porous media,

$$
\begin{aligned}
\frac{\partial}{\partial x_{i}} & {\left[\frac{k_{1 i j}}{\mu_{1}}\left(\frac{\partial p_{1}}{\partial x_{j}}+\rho_{1} g_{j}\right)+k_{1 T i j} \frac{\partial T_{1}}{\partial x_{j}}\right]=\left[n_{1} c_{1}+\left(\zeta_{2}-n_{2}\right)\left(c_{p}-c_{s}\right)\right.} \\
& \left.+\left(\zeta_{1}-n_{1}\right) c_{s}\right] \frac{\partial p_{1}}{\partial t}-\left(\zeta_{2}-n_{2}\right)\left(c_{p}-c_{s}\right) \frac{\partial p_{2}}{\partial t}+\zeta_{1} \frac{\partial^{2} u_{i}}{\partial t \partial x_{i}} \\
& -n_{1} c_{1 T} \frac{\partial T_{1}}{\partial t}-n_{1} c_{1 T} v_{1 i} \frac{\partial T_{1}}{\partial x_{i}}-\left(\zeta_{1}-n_{1}\right) c_{T} \frac{\partial T_{s}}{\partial t}+\Gamma \\
\frac{\partial}{\partial x_{i}} & {\left[\frac{k_{2 i j}}{\mu_{2}}\left(\frac{\partial p_{2}}{\partial x_{j}}+\rho_{2} g_{j}\right)+k_{2 T i j} \frac{\partial T_{2}}{\partial x_{j}}\right]=\left[n_{2} c_{2}+\left(\zeta_{2}-n_{2}\right) c_{p}\right] \frac{\partial p_{2}}{\partial t} } \\
& -\left(\zeta_{2}-n_{2}\right)\left(c_{p}-c_{s}\right) \frac{\partial p_{1}}{\partial t}+\zeta_{2} \frac{\partial^{2} u_{i}}{\partial t \partial x_{i}}-n_{2} c_{2 T} \frac{\partial T_{2}}{\partial t} \\
& -n_{2} c_{2 T} v_{1 i} \frac{\partial T_{2}}{\partial x_{i}}-\left(\zeta_{2}-n_{2}\right) c_{T} \frac{\partial T_{s}}{\partial t}-\Gamma
\end{aligned}
$$

\section{The Heat Transfer Model}

[10] Assuming the pore-fluid, fissure-fluid, and solid temperatures are not in local equilibrium, the energy balance equation for the solid phase can be written as,

$$
\begin{aligned}
\frac{\partial}{\partial x_{i}}\left(n_{s} \lambda_{s i j} \frac{\partial T_{s}}{\partial x_{j}}\right)= & n_{s} \rho_{s} C_{s}\left(\frac{\partial T_{s}}{\partial t}+v_{s i} \frac{\partial T_{s}}{\partial x_{i}}\right) \\
& -T_{s}\left(\zeta_{1}-n_{1}\right) c_{T} \frac{\partial p_{1}}{\partial t}-T_{s}\left(\zeta_{2}-n_{2}\right) c_{T} \frac{\partial p_{2}}{\partial t} \\
& +T_{s} \gamma \frac{\partial^{2} u_{i}}{\partial t \partial x_{i}}+\kappa_{s 1}\left(T_{s}-T_{1}\right)+\kappa_{s 2}\left(T_{s}-T_{2}\right)
\end{aligned}
$$

in which $\lambda_{s i j}$ is the thermal conductivity of solid phase, $C_{S}$ is the heat capacity of the solid, $\kappa_{s 1}$ is the coefficient of heat exchange between the solid and the pore-fluid, and $\kappa_{s 2}$ is the coefficient of heat exchange between the solid and the fissure-fluid. The Equation (21) accounts for the flux of thermal energy due conduction and advection, the change in the internal energy of the solid phase due to a change in pore and fluid pressures, change in the internal energy due 
to volume change of the solid skeleton, and the exchange of energy between the solid phase and the pore-fluid and between the solid phase and the fissure-fluid. The energy balance equation for the pore-fluid is in turn written as,

$$
\begin{aligned}
\frac{\partial}{\partial x_{i}}\left(n_{1} \lambda_{1 i j} \frac{\partial T_{1}}{\partial x_{i}}\right)= & C_{1}\left(\frac{\partial n_{1} \rho_{1} T_{1}}{\partial t}+\frac{\partial v_{1 i} n_{1} \rho_{1} T_{1}}{\partial x_{i}}\right)+\left.n_{1} T_{1} \beta_{1} \frac{d_{1} \varepsilon_{1}}{d t}\right|_{T_{1}} \\
& +C_{1} T_{1} \rho_{1}\langle\Gamma\rangle-C_{2} T_{2} \rho_{2}\langle-\Gamma\rangle+\kappa_{1 s}\left(T_{1}-T_{s}\right) \\
& +\kappa_{12}\left(T_{1}-T_{2}\right)
\end{aligned}
$$

with

$$
\left.\frac{d_{1} \varepsilon_{1}}{d t}\right|_{T_{l}}=-\left.\frac{1}{\rho_{1}} \frac{d_{1} \rho_{1}}{d t}\right|_{T_{l}}=-c_{1} \frac{d_{1} p_{1}}{d t}
$$

in which $\lambda_{1 i j}$ and $C_{1}$ are the thermal conductivity and the heat capacity of the pore-fluid, respectively. $\kappa_{12}$ is the coefficient of heat exchange between the pore-fluid and the fissure-fluid, and $\beta_{1}=\partial p_{1} /\left.\partial T_{1}\right|_{\varepsilon_{1}}=c_{1 T} / c_{1}$. In Equation 22, \langle\rangle refers to the Clebsch-Macaulay singularity function. The third term on the right hand side represents the change in the internal energy due to change in the volume of pore-fluid, the fourth term represents the convective heat transfer due to fluid exchange between the porous blocks and the fissure network. Noting that

$$
\begin{aligned}
\frac{\partial n_{1} \rho_{1} T_{1}}{\partial t}+\frac{\partial v_{1 i} n_{1} \rho_{1} T_{1}}{\partial x_{i}}= & T_{1}\left(\frac{\partial n_{1} \rho_{1}}{\partial t}+\frac{\partial v_{1 i} n_{1} \rho_{1}}{\partial x_{i}}\right) \\
& +n_{1} \rho_{1}\left(\frac{\partial T_{1}}{\partial t}+v_{1 i} \frac{\partial T_{1}}{\partial x_{i}}\right)
\end{aligned}
$$

and using Equation 23, Equation 22 can be written as,

$$
\begin{aligned}
\frac{\partial}{\partial x_{i}}\left(n_{1} \lambda_{l i j} \frac{\partial T_{1}}{\partial x_{i}}\right)= & n_{1} C_{1} \rho_{1} \frac{\partial T_{1}}{\partial t}-n_{1} c_{1 T} T_{1} \frac{\partial p_{1}}{\partial t}+C_{1} T_{1} \rho_{1}\langle\Gamma\rangle \\
& -C_{2} T_{2} \rho_{2}\langle-\Gamma\rangle+\kappa_{s 1}\left(T_{1}-T_{s}\right)+\kappa_{12}\left(T_{1}-T_{2}\right) \\
& +n_{1} C_{1} \rho_{1} v_{1 i} \frac{\partial T_{1}}{\partial x_{i}}
\end{aligned}
$$

Similarly, the energy balance equation for the fissure-fluid is written as,

$$
\begin{aligned}
\frac{\partial}{\partial x_{i}}\left(n_{2} \lambda_{2 i j} \frac{\partial T_{2}}{\partial x_{i}}\right)= & n_{2} C_{2} \rho_{2} \frac{\partial T_{2}}{\partial t}-n_{2} c_{2 T} T_{2} \frac{\partial p_{2}}{\partial t}-C_{1} T_{1} \rho_{1}\langle\Gamma\rangle \\
& +C_{2} T_{2} \rho_{2}\langle-\Gamma\rangle+\kappa_{s 2}\left(T_{2}-T_{s}\right) \\
& +\kappa_{12}\left(T_{2}-T_{1}\right)+n_{2} C_{2} \rho_{2} v_{2 i} \frac{\partial T_{2}}{\partial x_{i}}
\end{aligned}
$$

in which $\lambda_{2 i j}$ and $C_{2}$ are the thermal conductivity and the heat capacity of the fissure-fluid, respectively. Notice that in Equations 23 to 26 the term $C_{m} T_{m}$ could be replaced by the enthalpy, $h_{m}$.

\section{A Summary of the Governing Equations}

[11] In summary, the partial differential equations describing the coupled THM processes in an elastic medium with double porosity can be written as,

\section{Deformation}

$$
\begin{aligned}
G \frac{\partial^{2} u_{i}}{\partial x_{j} \partial x_{j}}+ & (\lambda+G) \frac{\partial^{2} u_{j}}{\partial x_{i} \partial x_{j}}-\zeta_{1} \frac{\partial p_{1}}{\partial x_{i}}-\zeta_{2} \frac{\partial p_{2}}{\partial x_{i}}-a_{\varepsilon T_{s}} \frac{\partial T_{s}}{\partial x_{i}} \\
& +F_{i}=0
\end{aligned}
$$

Fluid Flow

$$
\begin{aligned}
\frac{\partial}{\partial x_{i}} \frac{k_{1 i j}}{\mu_{1}}\left(\frac{\partial p_{1}}{\partial x_{j}}+\rho_{1} g_{j}\right)+\frac{\partial}{\partial x_{i}} k_{1 T i j} \frac{\partial T_{1}}{\partial x_{j}}= & a_{11} \frac{\partial p_{1}}{\partial t}-a_{12} \frac{\partial p_{2}}{\partial t}+\zeta_{1} \frac{\partial^{2} u_{i}}{\partial t \partial x_{i}} \\
& -a_{1 T_{s}} \frac{\partial T_{s}}{\partial t}-a_{1 T_{1}} \frac{\partial T_{1}}{\partial t} \\
& -n_{1} c_{1 T} v_{1 i} \frac{\partial T_{1}}{\partial x_{i}}+\Gamma
\end{aligned}
$$

$$
\begin{aligned}
\frac{k_{2 i j}}{\mu_{2}}\left(\frac{\partial p_{2}}{\partial x_{j}}+\rho_{2} g_{j}\right)+\frac{\partial}{\partial x_{i}} k_{2 T i j} \frac{\partial T_{2}}{\partial x_{j}}= & -a_{21} \frac{\partial p_{1}}{\partial t}+a_{22} \frac{\partial p_{2}}{\partial t}+\zeta_{2} \frac{\partial^{2} u_{i}}{\partial t \partial x_{i}} \\
& -a_{2 T_{s}} \frac{\partial T_{s}}{\partial t}-a_{2 T_{2}} \frac{\partial T_{2}}{\partial t} \\
& -n_{2} c_{2 T} v_{1 i} \frac{\partial T_{2}}{\partial x_{i}}-\Gamma
\end{aligned}
$$

Heat Transport

$$
\begin{aligned}
\frac{1}{T_{s}} \frac{\partial}{\partial x_{i}}\left(n_{s} \lambda_{s i j} \frac{\partial T_{s}}{\partial x_{j}}\right)= & a_{T_{s} T_{s}} \frac{\partial T_{s}}{d t}-a_{T_{s} 1} \frac{\partial p_{1}}{\partial t}-a_{T_{s} 2} \frac{\partial p_{2}}{\partial t}+a_{T_{s} \varepsilon} \frac{\partial^{2} u_{i}}{\partial t \partial x_{i}} \\
& +\frac{\kappa_{s 1}}{T_{s}}\left(T_{s}-T_{1}\right)+\frac{\kappa_{s 2}}{T_{s}}\left(T_{s}-T_{2}\right)+a_{T_{s} T_{s}} v_{s i} \frac{\partial T_{s}}{\partial x_{i}}
\end{aligned}
$$

$$
\begin{aligned}
\frac{1}{T_{1}} \frac{\partial}{\partial x_{i}}\left(n_{1} \lambda_{1 i j} \frac{\partial T_{1}}{\partial x_{i}}\right)= & a_{T_{1} T_{1}} \frac{\partial T_{1}}{\partial t}-a_{T_{1}} \frac{\partial p_{1}}{\partial t}+C_{1} T_{1} \rho_{1}\langle\Gamma\rangle \\
& -C_{2} T_{2} \rho_{2}\langle-\Gamma\rangle+\frac{\kappa_{1 s}}{T_{1}}\left(T_{1}-T_{s}\right) \\
& +\frac{\kappa_{12}}{T_{1}}\left(T_{1}-T_{2}\right)+a_{T_{1} T_{1}} v_{1 i} \frac{\partial T_{1}}{\partial x_{i}}
\end{aligned}
$$

$$
\begin{aligned}
\frac{1}{T_{2}} \frac{\partial}{\partial x_{i}}\left(n_{2} \lambda_{2 i j} \frac{\partial T_{2}}{\partial x_{i}}\right)= & a_{T_{2} T_{2}} \frac{\partial T_{2}}{\partial t}-a_{T_{2} 2} \frac{\partial p_{2}}{\partial t}-C_{1} T_{1} \rho_{1}\langle\Gamma\rangle \\
& +C_{2} T_{2} \rho_{2}\langle-\Gamma\rangle+\frac{\kappa_{2 s}}{T_{2}}\left(T_{2}-T_{s}\right) \\
& +\frac{\kappa_{21}}{T_{2}}\left(T_{2}-T_{1}\right)+a_{T_{2} T_{2}} v_{2 i} \frac{\partial T_{1}}{\partial x_{i}}
\end{aligned}
$$

subject to the constitutive equations,

$$
\begin{aligned}
\frac{d \sigma_{i i}}{3} & =c^{-1} d \varepsilon_{k k}-\zeta_{1} d p_{1}-\zeta_{2} d p_{2}-a_{\varepsilon T_{s}} d T_{s} \\
\frac{d m_{1}}{\rho_{1 o}} & =\zeta_{1} d \varepsilon_{i i}+a_{11} d p_{1}-a_{12} d p_{2}-a_{1 T_{s}} d T_{s}-a_{1 T_{1}} d T_{1} \\
\frac{d m_{2}}{\rho_{2 o}} & =\zeta_{2} d \varepsilon_{i i}-a_{21} d p_{1}+a_{22} d p_{2}-a_{2 T_{s}} d T_{s}-a_{2 T_{2}} d T_{2} \\
d s_{s} & =a_{T_{s} \varepsilon} d \varepsilon_{i i}-a_{T_{s}} d p_{1}-a_{T_{s} 2} d p_{2}+a_{T_{s} T_{s}} d T_{s} \\
d s_{1} & =-a_{T_{1} 1} d p_{1}+a_{T_{1} T_{1}} d T_{1} \\
d s_{2} & =-a_{T_{2} 2} d p_{2}+a_{T_{2} T_{2}} d T_{2} ; \Gamma=\eta\left(p_{1}-p_{2}\right)
\end{aligned}
$$


with

$$
\begin{aligned}
\zeta_{1} & =\frac{c_{p}}{c}-\frac{c_{s}}{c}, \zeta_{2}=1-\frac{c_{p}}{c} \\
a_{11} & =n_{1} c_{1}+a_{12}+\left(\zeta_{1}-n_{1}\right) c_{s}, a_{22}=n_{2} c_{2}+a_{12}+\left(\zeta_{2}-n_{2}\right) c_{s}, \\
a_{12} & =a_{21}=\left(\zeta_{2}-n_{2}\right)\left(c_{p}-c_{s}\right), \\
a_{\varepsilon} & =a_{T_{s} \varepsilon}=\gamma, a_{1 T_{l}}=a_{T_{l} 1}=n_{1} c_{1 T}, \\
a_{2 T_{2}} & =a_{T_{2} 2}=n_{2} c_{2 T}, a_{1 T_{s}}=a_{T_{s} 1}=\left(\zeta_{1}-n_{1}\right) c_{T}, \\
a_{2 T_{s}} & =a_{T_{s} 2}=\left(\zeta_{2}-n_{2}\right) c_{T}, a_{T_{s} T_{s}}=\frac{n_{s} \rho_{s} C_{s}}{T_{s}}, \\
a_{T_{1} T_{1 l}} & =\frac{n_{1} \rho_{1} C_{1}}{T_{1}}, a_{T_{2} T_{2}}=\frac{n_{2} C_{2} \rho_{2}}{T_{2}}
\end{aligned}
$$

where $m_{1}$ and $m_{2}$ are the mass pore-fluid and mass fissure fluid per reference volume, respectively. $\rho_{1 o}$ and $\rho_{2 o}$ are the densities of the pore and fissure fluid at the reference configuration, respectively. Similarly, $s_{s}, s_{1}$, and $s_{2}$ are the entropies of solid, pore fluid and fissure fluid, respectively. The constitutive equations in (28) enjoy the major symmetry due to the reversibility of the physical processes taken into account. This result can also be achieved using Biot's free energy approach, within the general framework of mixture theory.

\section{Conclusions}

[12] It is shown that the development of the equations governing THM processes in elastic media with double porosity can be approached in a systematic manner where all the constitutive equations governing deformability, fluid flow and heat transfer are combined with the relevant conservation laws. The double porosity nature of the medium requires the introduction of dependent variables applicable to the deformable solid, and the fluid phases in the two void spaces. The governing partial differential equations are linear in view of the linearized forms of the constitutive assumptions invoked in the formulations. The linearity of these governing equations makes them amenable to solution through conventional mathematical techniques applicable to the study of initial boundary value problems in mathematical physics [Selvadurai, 2000]. Such solutions should serve as benchmarks for appropriate computational developments.

\section{References}

Bai, M., and J. C. Roegiers, Fluid flow and heat flow in deformable fractured porous media, Int. J. Engng Sci., 32(10), 1615-1633, 1994.

Callari, C., and F. Federico, FEM validation of a double porosity elastic model for consolidation of structurally complex clayey soils, Int. J. Numer. Analy. Meth., 24(4), 367-402, 2000.

Khalili, N., and S. Valliappan, Unified theory of flow and deformation in double porous media, European Journal of Mechanics, A/Solids., 15(2), 321-336, 1996.

Loret, B., and E. Rizzi, Strain localisation in fluid saturated anisotropic elastic plastic porous media with double porosity, J. Mech. Phys. Solids., 47, 503-530, 1999

Masters, I., W. K. S. Pao, and R. W. Lewis, Coupling temperature to a double-porosity model of deformable porous media, Int. J. Numerical Methods in Engineering, 49(3), 421-438, 2000.

Pao, W. K. S., and R. W. Lewis, Three-dimensional finite element simulation of three-phase flow in a deforming fissured reservoir, Comput. Methods. Appl. M., 191(23-24), 2631-2659, 2002.

Selvadurai, A. P. S., Partial Differential Equations in Mechanics, Vols. 1 and 2, Springer-Verlag, Berlin, 2000.

N. Khalili, School of Civil and Environmental Engineering, The University of New South Wales, Sydney, 2052, Australia. (n.khalili@unsw. edu.au)

A. P. S. Selvadurai, Department of Civil Engineering and Applied Mechanics, McGill University, Montreal, QC, Canada, H3A 2K6. 